Easy, Efficient, and Mobile Way to Train Microsurgical Skills

During Busy Life of Neurosurgical Residency in

Resource-Challenged Environment

\author{
Huotarinen, Antti
}

2017-11

Huotarinen, A , Niemela , M \& Jahromi , B R 2017 , ' Easy, Efficient, and Mobile Way to

Train Microsurgical Skills During Busy Life of Neurosurgical Residency in

Resource-Challenged Environment ' , World Neurosurgery , vol. 107 , pp. 358-361 . https://doi.org/10.1016/j.wneu.2

http://hdl.handle.net/10138/298154

https://doi.org/10.1016/j.wneu.2017.08.024

publishedVersion

Downloaded from Helda, University of Helsinki institutional repository.

This is an electronic reprint of the original article.

This reprint may differ from the original in pagination and typographic detail.

Please cite the original version. 


\section{Easy, Efficient, and Mobile Way to Train Microsurgical Skills During Busy Life of Neurosurgical Residency in Resource-Challenged Environment}

\section{Antti Huotarinen, Mika Niemelä, Behnam Rezai Jahromi}

BACKGROUND: Residents" lives are hectic-it is hard to find a place and time for training basic and advanced microsurgical skills. Surgical instruments and sutures can be purchased (or loaned from another department), but the most expensive and space-occupying device is the microscope. In developing countries, microscopes are used where they are needed most, in operating rooms. Furthermore, a conventional microscope is not portable. For all of these reasons, the availability of microscopes for training microsurgery is limited.

METHOD: We used a coffee cup and smartphone (CCS) as a training device instead of a microscope. The coffee cup was the base, and the smartphone functioned to magnify, illuminate, and visualize objects. We measured 2 residents' performance on end-to-end artificial bypass before and after 5 days of CCS-based training.

RESULTS: We were able to quickly set up the environment for practicing microsurgical skills in any surrounding. After 5 days of training with CCS we could see significant development of microsurgical performance with a conventional microscope as well. The learning curve was dependent on baseline performance.

CONCLUSION: CCS is efficient, mobile, and easy to set up. Even though our smartphone-based training was in 2 dimensions, we could improve our microsurgical performance with conventional microscopes, which have 3-dimensional capability. CCS also provides an easy method to record one's microsurgical training. CCS improved both of the subjects' microsurgical performance, making it a good alternative for a traditional microscope.

\footnotetext{
Key words

- Developing countries

- Microsurgery

- Residency

- Smartphone

- Training
}

Abbreviations

CCS: Coffee cup and smartphone

E-E: End-to-end (bypass)

\section{INTRODUCTION}

$1 \sqrt{ }$ any neurosurgical residents have difficulty finding opportunities to learn and practice microsurgical skills due to lack of time, space, or equipment. One of the most important factors of being a good neurosurgeon is controlling micromovements. A significant amount of time and practice is necessary for achieving high-level microsurgical skills. Making the microsurgical training environment more available for trainees would provide the opportunity to practice more often. Conventional microscopes are large and immobile, limiting microsurgical training to a predefined location. In developing countries, ${ }^{\mathrm{I}}$ limited resources are used for patient care and it is hard to justify the purchase of an expensive microscope for training use only.

Fortunately, smartphones are now widely used globally $->$ I billion of them have been sold. Built-in cameras have high-quality lenses, zooming qualities, and video recording capability. Smartphone screens have high-density pixels, making images of videos good. These qualities were found only in professional cameras just a few years ago. The main causes for using a microscope for microsurgery are magnification and illumination. Today's smartphones have both of these qualities. Our aim was to construct and test a minimalist microsurgical training platform that would be easy to set up and use practically anywhere. ${ }^{2,3}$

\section{MATERIAL AND METHODS}

We used a coffee cup and smartphone (CCS) instead of a microscope (Figure 1). Two different phones (iPhone 6 and iPhone 7 Plus, Apple, Cupertino, California, USA) were tested during our task. Different coffee cup sizes can be used to enhance zoom and focus. The reason for using a coffee cup is to show that any available "body" for a smartphone is possible for training. ${ }^{4}$ The coffee cup acted as a stand for the smartphone. The smartphone camera was activated, and the flash and zoom features were used. With this simple method we were able to produce an environment for microsurgical training by using only a CCS.
Department of Neurosurgery, Helsinki University Hospital, Clinical Neurosciences,

Neurosurgery, University of Helsinki, Helsinki, Finland

To whom correspondence should be addressed: Antti Huotarinen, M.D.

[E-mail: antti.huotarinen@hus.fi]

Citation: World Neurosurg. (2017) 107:358-361

http://dx.doi.org/10.1016/j.wneu.2017.08.024

Journal homepage: www.WORLDNEUROSURGERY.org

Available online: www.sciencedirect.com

1878-8750/\$ - see front matter (c) 2017 Elsevier Inc. All rights reserved. 

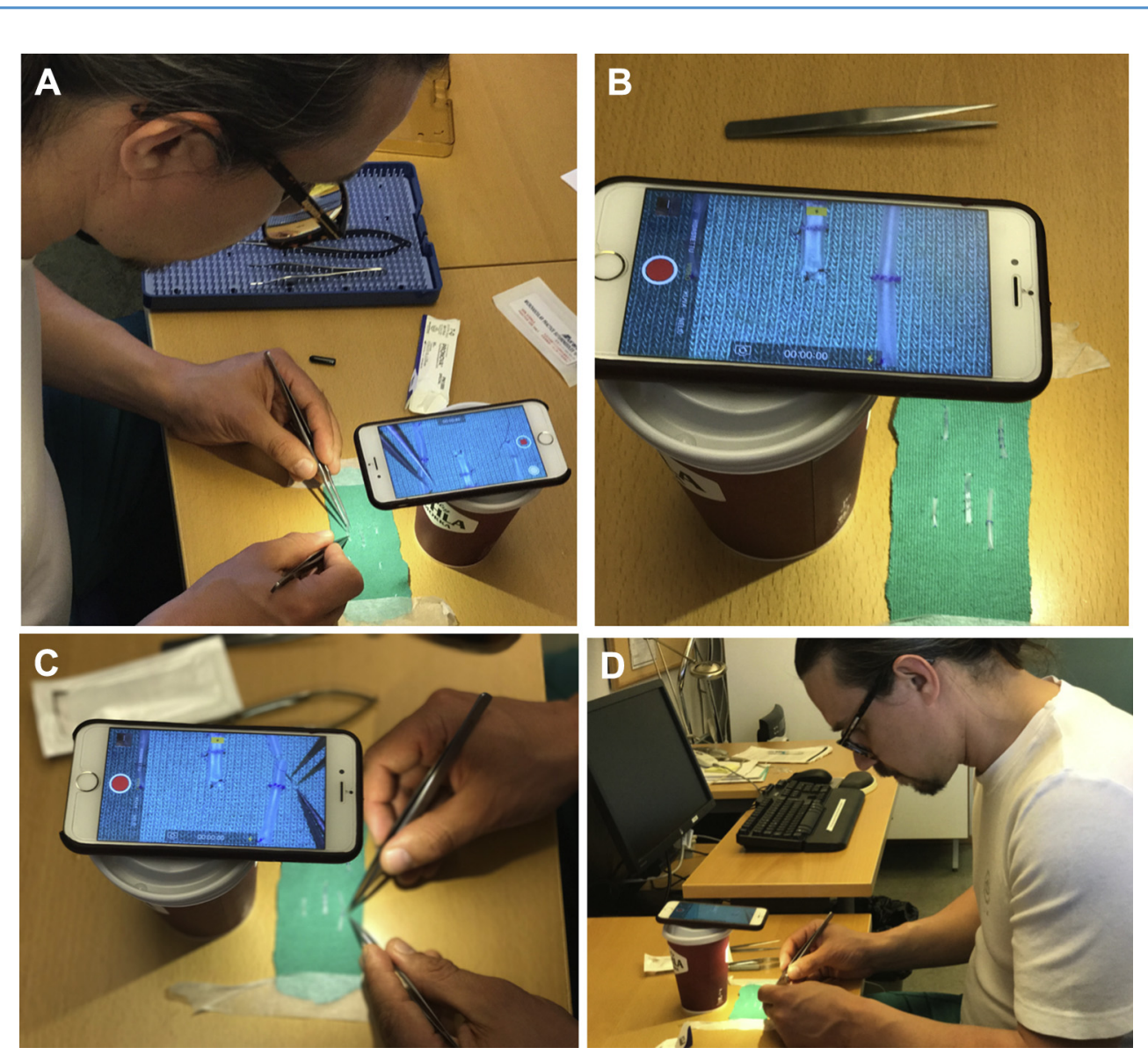

Figure 1. (A) All equipment needed for training is seen: coffee cup, smartphone, sutures, and instruments. Any table works as a training area. (B) If the smartphone zoom or focus is limited, one can try to change cup size

to rearrange the focusing and zoom area. (C) Recording of training is possible with a smartphone. (D) Hands are free to work while seeing one's own movements via the smartphone screen.

Suitable suture sizes from 6-o to 9-o were tested under the CCS platform by making sutures on a $4-\mathrm{mm}$ silicone tube. Baseline skill measurement was conducted with a Pentero microscope (Zeiss, Oberkochen, Germany) with $6 \times$ magnification.

\section{Study Design}

Baseline performance was measured by a training set of $4-5$ endto-end (E-E) bypasses on a 4-mm silicone tube with 7-o sutures under microscope. For 5 days both trainees worked with the CCS concept and trained under a structural program, performing daily training sets consisting of either 4 E-E bypasses and/or a similar amount of interrupted suturing on a glove with 7-o thread. On the last day of the study, we repeated the baseline task of E-E bypasses under the microscope to monitor any improvement. For trainee I (at the end of residency), we recorded all the videos and calculated times for the individual practice bypasses and all individual sutures from video. For trainee 2 (just commencing residency), we recorded times needed to complete training sets of 5 bypasses. All training sets were done with the same personal training instruments and 7-o Premilene (B. Braun, Melsungen, Germany) 7-0 sutures, with I0-mm 3/8 needles cut to I2-cm lengths before each individual bypass. Video analysis was performed on Final Cut Pro software (Apple). Individual suture times were defined as time between consecutive sutures as the needle was picked up. The numbers of completed sutures were verified from video.

\section{RESULTS}

CCS proved to be an excellent training platform for improving microsurgical skills. The setting up of CCS practice took $2-5$ minutes with instruments available. The setup for microscope practice in an empty operating room took 17 minutes. We were able to suture up to 9-o thread relatively easily with the newer and larger smartphone while this task was slower with the older, smaller phone. The CCS provided a 2-dimensional image, which needed some adjustment for the trainee because of a loss of sense of depth.

\section{Trainee 1}

The time needed for an individual suture under the microscope improved between the initial baseline training set from 96.2 
seconds $(\mathfrak{n}=40,95 \%$ confidence interval $[\mathrm{CI}] 84.34-$ I08.I seconds) to 8 I. 73 seconds ( $n=55,95 \%$ CI 74.92-88.54 seconds) during the final training set (2-tailed Student's t-test $\mathrm{P}=0.0258)$. The time needed for individual sutures with CCS improved from I8I.3 seconds ( $n=43,95 \%$ CI I63.0-I99.5 seconds) to I32.5 seconds ( $n=43,95 \%$ CI 74.92-88.54 seconds) (2-tailed Student's t-test $\mathrm{P}<\mathrm{0.000I}$ ) (Figure 2A). At baseline, the average time for $\mathrm{E}-\mathrm{E}$ bypass was I6.03 minutes (standard deviation [SD] 3.049, mean Io.o sutures) with microscope and 27.07 minutes (SD 4.286, mean 9.5 sutures) for CCS. At the final training set the average time for end-to-bypass was $18.73 \mathrm{~min}$ (S.D. 0.78I4, mean $\mathrm{I3} .75$ sutures) with microscope and 19.4 minutes (SD I.I57, mean I0.75 sutures) with CCS. There was no significant difference in bypass time between baseline microscope and final microscope training sets for bypass time (2-tailed Student's t-test $\mathrm{P}=0.137$ ), but CCSr bypass times improved statistically significantly (2-tailed Student's t-test $\mathrm{P}=$ o.0136) (Figure 2C). The number of sutures placed under the microscope improved significantly (2-tailed Student's $t$-test $\mathrm{P}=$ o.oor), (Figure 2B).

\section{Trainee 2}

The time needed for a 5 E-E bypass training set improved from 2 hours and 40 minutes to I hour and 54 minutes. The learning curve of trainee 2 was more radical because he was a beginner in microsurgery.

CCS gave additional cognitive stress since the task was more difficult to perform compared with a microscope. However, even short periods of structural training diminished these difficulties and compensatory techniques were found. The manipulation of instruments with $2-\mathrm{D}$ visualization seemed to rely more on proprioceptive and haptic feedback when compared with 3-D capable microscopes.

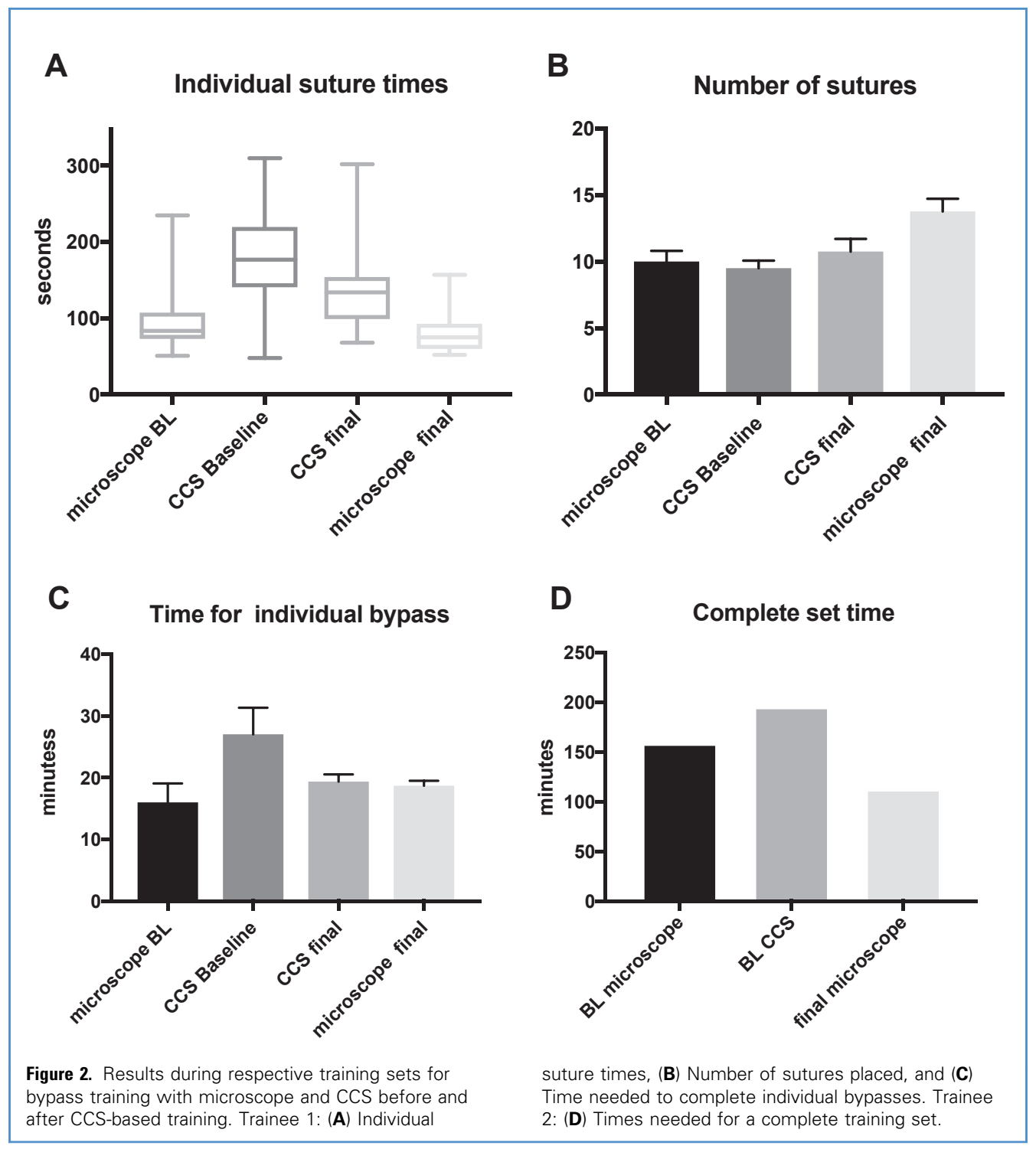


Equipment

Image quality can be improved by investing in a better-equipped camera phone. The background color of the working area can be easily changed to suit suture color and ambient light for better contrast. For enhancing visual field and zooming, we tried different commercial applications. However, we noticed that the standard smartphone camera application proved to be both reliable and adequate.

\section{CONCLUSION}

CCS is an excellent and accessible option for practicing microsurgical skills. Our data show that smartphone-based training can also improve microsurgical performance under the microscope, at least in short-term practice. It provides an easy means to challenge oneself by recording and comparing one's development of skills over time. CCS also provides a good opportunity to use widely available technology for microsurgical training in developing countries while preserving limited resources for patient care.

We believe that this simple method can bring mobile microscopy for residents. With all the difficulties of finding an appropriate training place, microscope, and time within the hospital, CCS-based training can overcome these limitations.

\section{REFERENCES}

I. Kaplan WA. Can the ubiquitous power of mobile phones be used to improve health outcomes in developing countries? Global Health. 2006;2:9.

2. Ozdalga E, Ark O, Neera A. The smartphone in medicine: a review of current and potential use among physicians and students. J Med Internet Res. 20I2;I4:eI28.
3. Poushter J. Smartphone Ownership and Internet Usage Continues to Climb in Emerging Economies. Washington, DC, USA: Pew Research Center; 2016.

4. Kim DM, Kang JW, Kim JK, Youn I, Park JW. Microsurgery training using a smartphone. Microsurgery. 2015;35:500-50I.

Conflict of interest statement: No funding was received for this study, and all authors denied a conflict of interest.

Received 26 May 2017; accepted 1 August 2017
Citation: World Neurosurg. (2017) 107:358-361. http://dx.doi.org/10.1016/j.wneu.2017.08.024 Journal homepage: www.WORLDNEUROSURGERY.org Available online: www.sciencedirect.com 1878-8750/\$ - see front matter (c) 2017 Elsevier Inc. All rights reserved. 